\title{
Autonomic healable waterborne organic-inorganic polyurethane hybrids based on aromatic disulfide moieties
}

\author{
R. H. Aguirresarobe ${ }^{1}$, L. Martin $^{2}$, M. J. Fernandez-Berridi ${ }^{1}$, L. Irusta ${ }^{1 *}$ \\ ${ }^{1}$ POLYMAT, Polymer Science and Technology Department, Faculty of Chemistry, University of the Basque Country, \\ UPV/EHU, Spain \\ ${ }^{2}$ Macrobehavior-Mesostructure-Nanotechnology SGiker service of UPV/EHU, Polytechnic School, UPV/EHU, Spain
}

Received 14 September 2016; accepted in revised form 16 November 2016

\begin{abstract}
Aromatic disulfide dynamic structures were incorporated as chain extenders in waterborne organic-inorganic polyurethane hybrids in order to provide autonomic healable characteristics. The synthesis was carried out following the acetone process methodology and the influence of the introduction of the healing agents in the polymer dispersion stability was analyzed. After the crosslinking process at room temperature, organic-inorganic hybrid films, which presented autonomic healing characteristics, were obtained. These features were evaluated by means of stress-strain tests and the films showed repetitive healing abilities. Thus, the optimum healing time at room temperature $\left(25^{\circ} \mathrm{C}\right)$ as well as the influence of different parameters in the healing efficiency, such the aromatic disulfide concentration or the physical properties of the polymer matrix were analyzed.
\end{abstract}

Keywords: smart polymers, metathesis, waterborne polyurethanes, disulfide bonds, healing

\section{Introduction}

The incorporation of healable characteristics in commercial polymers has been a topic of interest in the last decade, as the ability to recover the physical properties of a damaged material potentially permits the development of smart applications that make materials safer, more reliable and durable and reduce maintenance costs [1-4]. However, the incorporation of such characteristics, by means of the introduction of healing agents, can negatively affect the physical properties of the final product. Similarly, the different functionalities presented in the polymer backbone could interfere in the healing process. As a result, few studies have been reported for complex formulations, such as adhesives or coatings, where healing properties are highly desirable.

The key challenge is to introduce healing agents with specific interactions, avoiding undesirable reactions with other components and/or functionalities presented in the polymer structure while providing highly efficient reparations. Thus, the different components of the formulation should be thoroughly selected taking into account not only the intrinsic properties of the final material and the characteristics of the reparation mechanism but also the possible interactions among the different components, both in the polymerization process and during the life of the material. This work assesses the possibility of incorporating healing characteristics to waterborne polyurethanes, offering alternatives to develop environmentally friendly healable materials able to be applied as films or coatings for automobiles, textiles, furniture and wood. Among the different healing alternatives reported in literature, disulfide metathesis was selected as the reaction to provide healing characteristics to our waterborne polyurethane structures. This dynamic

$\overline{{ }^{*} \text { Corresponding author, e-mail: lourdes.irusta@ehu.eus }}$ (C) BME-PT 
reaction is known to provoke the exchange of the side groups of the disulfide bridges in the presence of initiators or catalysts [5-8]. Once incorporated in the polymer network, these structures are capable of breaking and reforming linkages, and, as a consequence, these materials act as vitrimers, that is, solid polymers during their lifetime that under certain conditions can flow and thereby promote material healing [2, 9-12].

The capability of different disulfides to undergo disulfide metathesis reactions mainly depends on their chemical structures. Thus, while aliphatic disulfide structures require an external energy source, such as high temperatures or UV light [13-17] to trigger the reaction, aromatic disulfide moieties can chain exchange under mild conditions, even at room temperature, without a catalyst [18-20]. Recently, taking advantage of these properties, aromatic disulfide structures have been incorporated in order to develop autonomic healable polyurethanes, opening new alternatives in the field of self-healing materials [21-23]. The concept has been applied to cross-linked polyurehanes, where metathesis of the disulfide linkages permits the reparation of fractured elastomers. However, owing to the characteristics of the disulfide moieties, these structures can interact with other functionalities presented in the formulation. Thus, the high reactivity of disulfide structures against free radicals can inhibit the free radical polymerization processes, commonly used in the crosslinking of acrylate modified waterborne polyurethanes.

To overcome this drawback, we have selected organic-inorganic hybrid polyurethanes to develop our waterborne healable materials. These materials incorporate inorganic precursors, such as alkoxysilanes, which lead to material crosslinking by condensation reactions [24]. The synthesis of these materials should be precisely controlled as slight modifications in the formulation or reaction conditions can provoke polymer precipitation $[25,26]$. In addition, the incorporation of inorganic precursors influences the material properties, increasing the modulus and the resistance of the material but reducing their ductility [27]. Therefore, the effect of different functionalities on the healing process as well as the influence of the incorporation of healing agents in the water dispersion stability and crosslinking abilities of the final material should be considered.

The proposed organic-inorganic polyurethane structure incorporates aromatic disulfide moieties as chain extenders, using 4-aminophenyl disulfide (APDS) as monomer and N-butyl-3-aminopropyl trimethoxysilane (N-bAPTMS) in both chain ends of the polyurethane structure as alkoxysilane precursors. The condensation of these precursors gives rise to siliconoxygen networks and consequently to hybrid formation. In the solid state, the dynamic chain exchange reactions of disulfide bonds would enable the recovery of the structural integrity of the material after being damaged.

\section{Experimental section}

\subsection{Materials}

Isophorone diisocyanate (IPDI), 2-bis(hydroxymethyl) propionic acid (DMPA), polypropylene glycol (PPG) $\left(M_{\mathrm{n}} 1000 \mathrm{~g} \cdot \mathrm{mol}^{-1}\right)$, triethylamine (TEA), dibutyltin diacetate (DBTDA), acetone, 4-Aminophenyl disulfide (APDS) and 4,4'-ethylenedianiline (APDM) were purchased from Sigma-Aldrich Chemical Corporation (Steinheim, Germany). N-butyl-3-aminopropyl trimethoxysilane (N-bAPTMS) was purchased from Fluorochem Ltd. (Hadfield, UK). All materials were used as received.

\subsection{Synthesis of waterborne polyurethane}

Waterborne polyurethanes were synthesized according to the so called 'acetone process' [28-30]. This methodology consists of the synthesis of the polyurethane structure in a low boiling point solvent, usually acetone, incorporating hydrophilic groups into the polymer backbone in order to act as internal emulsifiers. In a second step, water is added in a controlled way and finally, acetone is evaporated in order to obtain the final waterborne polyurethane dispersion. The synthetic way to obtain PPGAPDS polymer is shown in Figure 1.

PPG, the internal emulsifier (DMPA) and TEA were poured together with DBTDA (800 ppm) and acetone $(36 \mathrm{~g})$ into a flask reactor. When the reaction temperature reached $56^{\circ} \mathrm{C}$, IPDI was added and the reaction was maintained for $3 \mathrm{~h}$. In a further step, the reaction mixture was cooled down to room temperature and the polymer chains were extended using the appropriate APDS amount, leaving some free isocyanate groups. This reaction was carried out for one additional hour. Finally, N-bAPTMS was added in a stoichiometric ratio in order to react with the remaining isocyanate groups, end-capping the polymer chains with the alkoxysilane precursors. In addition to APDS based waterborne polyurethanes, polymer 


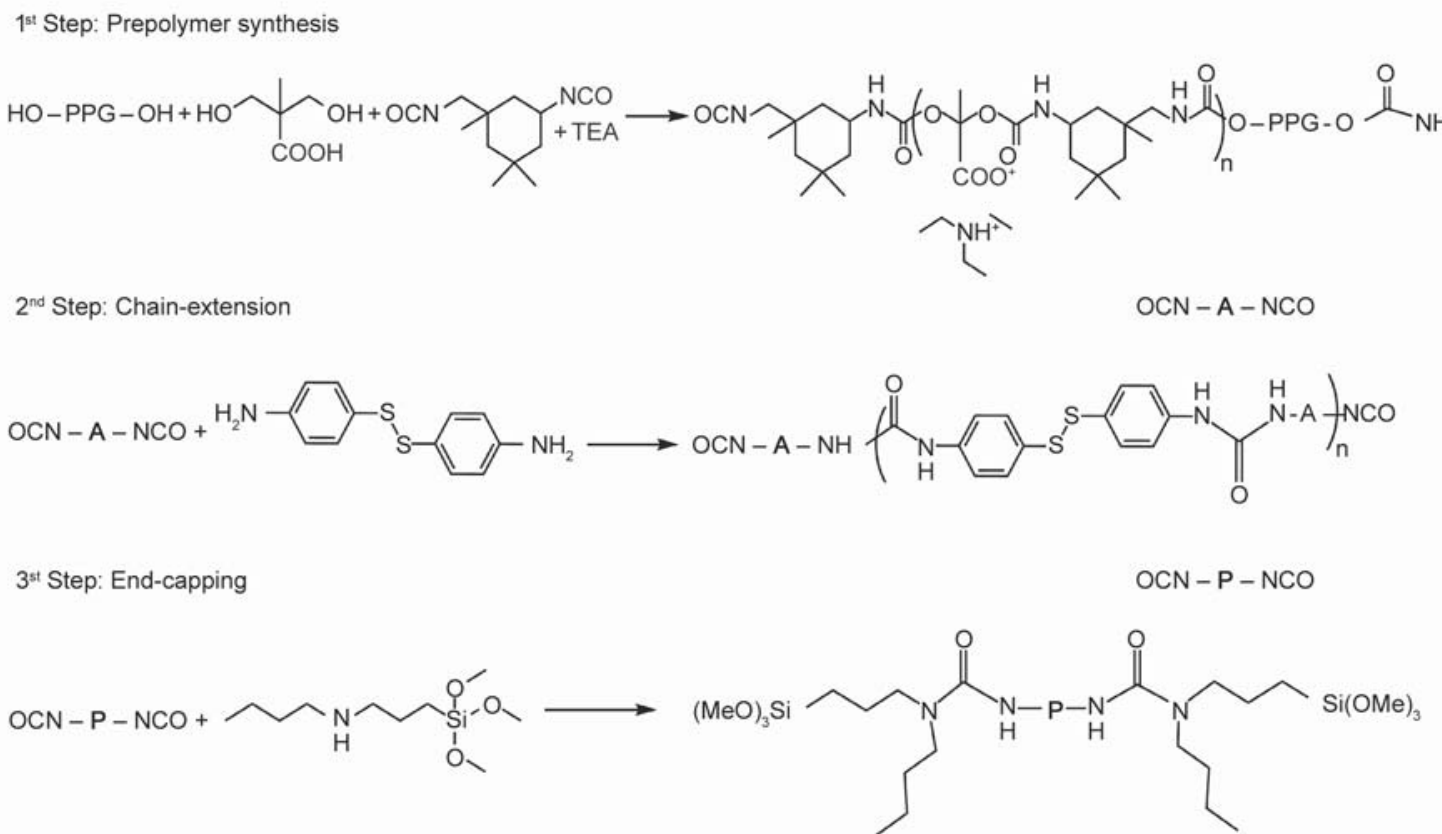

Figure 1. Synthetic pathway to obtain waterborne PPGAPDS polyurethanes

Table 1. Amount of reagents used in the synthesis of different PPGAPDS and PPGAPDM samples

\begin{tabular}{|c|c|c|c|c|c|c|c|c|}
\hline \multirow{2}{*}{ Sample } & PPG & DMPA & IPDI & TEA & APDS & N-bAPTMS & APDS & N-bAPTMS \\
\hline & \multicolumn{6}{|c|}{ [mmol] } & \multicolumn{2}{|c|}{$\left[w t^{\circ} \%\right]$} \\
\hline PPG2APDS & 22.5 & 11 & 56.5 & 15 & 3.0 & 40 & 2.2 & 20.1 \\
\hline PPG5APDS & 22.5 & 11 & 56.5 & 15 & 7.0 & 32 & 5.2 & 16.4 \\
\hline PPG10APDS & 22.5 & 11 & 56.5 & 15 & 18.5 & 20 & 10.1 & 10.6 \\
\hline PPG15APDS & 22.5 & 11 & 56.5 & 15 & 23.0 & 9 & 15.0 & 4.9 \\
\hline \multicolumn{9}{|c|}{ APDM } \\
\hline PPG5APDM & 22.5 & 11 & 56.5 & 15 & 7.0 & 32 & 5.0 & 16.5 \\
\hline PPG10APDM & 22.5 & 11 & 56.5 & 15 & 18.5 & 20 & 10.1 & 10.6 \\
\hline
\end{tabular}

structures containing 4,4'-ethylenedianiline (APDM) were synthesized under similar reaction conditions by replacing the APDS monomer. Table 1 summarizes the formulations used in the different reactions. As shown in Table 1, the amount of N-APTMS was reduced when increasing the amount of disulfide moieties in the sample. As a consequence, the soft/hard segment ratio and therefore the percentage of urethane groups was maintained constant for all formulations.

\subsection{Instrumentation}

Fourier Transform Infrared (FTIR) spectra of the synthesized polymers were performed in a Nicolet 6700 spectrometer (Thermo Scientific, Madison, WI, USA) at a resolution of $4 \mathrm{~cm}^{-1}$ and a total of 10 interferograms were signal averaged. ${ }^{1} \mathrm{H}$ liquid Nuclear Magnetic Resonance (NMR) spectra were obtained in a Fourier Transform Bruker $300 \mathrm{MHz}$ spectrometer (model Avance 300 DPX, Billerica, MA, USA), while solid state ${ }^{29} \mathrm{Si}$ spectra were performed in a Bruker Avance II 400 spectrometer using cross polar- ization magic angle spinning methodology. Dynamic Light Scattering (DLS) measurements were carried out using a 90Plus (Brookhaven, Holstville, NY, USA) Particle Size Analyzer in order to obtain the diameter of the particles, $D_{\mathrm{p}}$, as an average of eight measurements. Tensile tests were carried out on a universal Instron 5569 (Norwood, MA, USA) tensile test machine. A load cell of $100 \mathrm{~N}$ was used and the initial distance between clamps was fixed at $30 \mathrm{~mm}$. The experiments were performed using a crossheadspeed of $20 \mathrm{~mm} / \mathrm{min}$. AFM images were obtained in a Dimension ICON Nanoscope V (Bruker, Billerica, MA, USA) device, using TESP-V2 tips $(f=320 \mathrm{kHz}$, $k=40 \mathrm{~N} / \mathrm{m})$. The images were obtained in $1 \mu \mathrm{m} \times 1 \mu \mathrm{m}$ frames.

\section{Results and discussion}

\subsection{Characterization}

The polymerization reaction of APDS and APDM based waterborne polyurethanes was followed by FTIR spectroscopy as presented in Figure 2. 

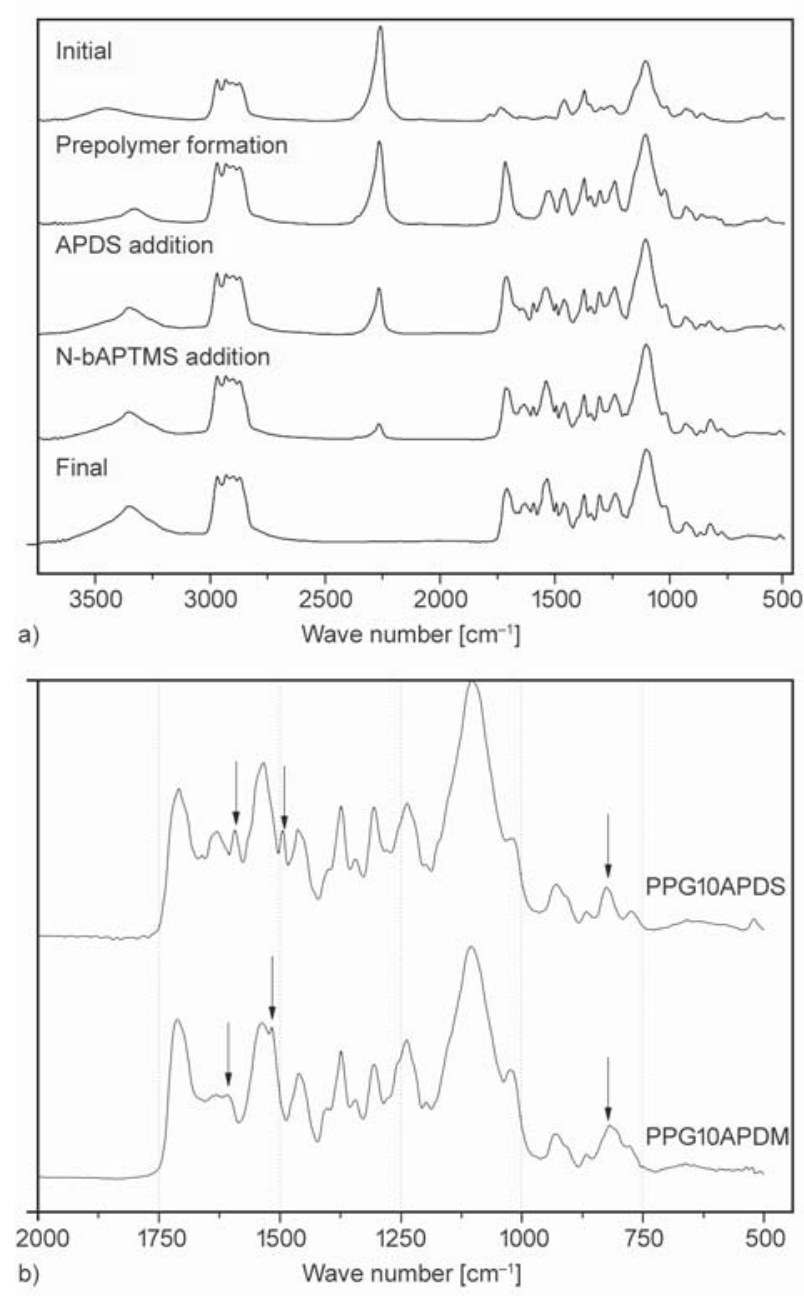

Figure 2. Evolution of the FTIR spectra in the different reaction steps for PPG10APDS sample (a) and scale expanded FTIR spectra for PPG10APDS and PPG10APDM (b)
As observed in Figure 2a, the main spectral features correspond to the isocyanate (stretching vibration at $2260 \mathrm{~cm}^{-1}$ ) and urethane groups (Amide I Amide II bands at $1735 \mathrm{~cm}-1$ and $1550 \mathrm{~cm}^{-1}$ as well as the $\mathrm{N}-\mathrm{H}$ stretching vibration at $3330 \mathrm{~cm}^{-1}$ ). When APDS (or APDM) is added, the bands corresponding to the $\mathrm{C}=\mathrm{C}$ stretching vibration of the aromatic rings (1595$1495 \mathrm{~cm}^{-1}$ ) are detected. Additionally, the Amide I band corresponding to urea bonds can be observed at $1635 \mathrm{~cm}^{-1}$. Finally, the N-bAPTMS addition increases the absorbance of the band due to the urea groups and a new band assigned to the $\mathrm{Si}-\mathrm{O}-\mathrm{R}$ bending vibration at $825 \mathrm{~cm}^{-1}$ appears. The final spectra are characterized by the disappearance of the isocyanate stretching vibration band, whereas the rest of the aforementioned spectral bands are maintained, as shown in Figure $2 b$. The structural characterization of the obtained polymers was studied by ${ }^{1} \mathrm{H}-\mathrm{NMR}$ spectroscopy. The ${ }^{1} \mathrm{H}-\mathrm{NMR}$ spectrum and the line assignment for PPG10APDS and PPG10APDM samples are shown in Figure 3.

The most interesting lines for PPGAPDS and PPGAPDM samples is the one at $2.8 \mathrm{ppm}$, which corresponds to the urea linkages, and those at 6.5 and $7.2 \mathrm{ppm}$ due to the aromatic ring protons. In addition, for PPG10APDM samples, the line corresponding to methylene groups of $4{ }^{\prime} 4$ - ethylenedianiline residue was also observed. Thus, according to the proposed line assignment for both systems, the incorporation of APDS (or APDM) and N-bAPTMS

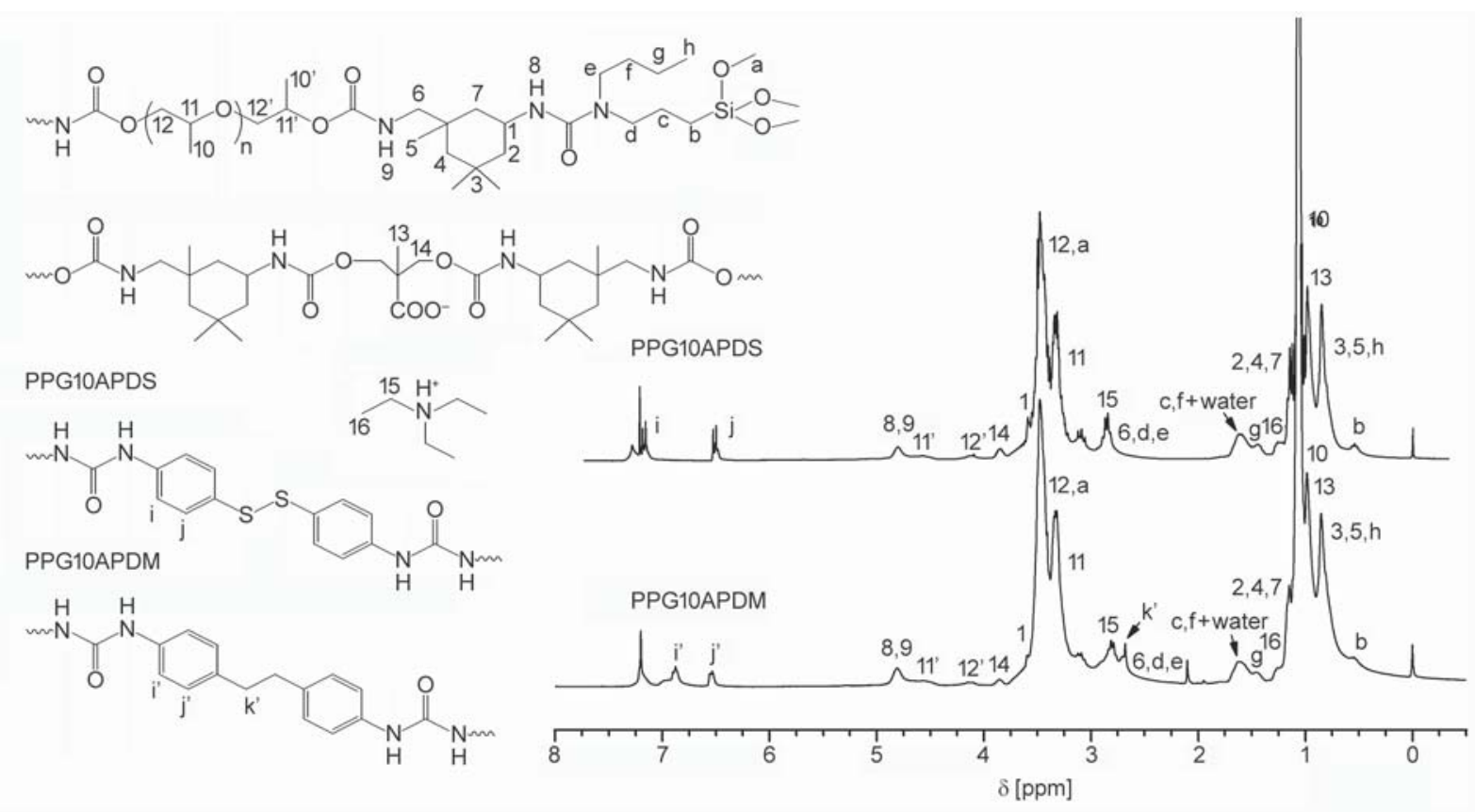

Figure 3. ${ }^{1} \mathrm{H}$ NMR and line assignment of PPG10APDS and PPG10APDM systems 
Table 2. Particle size of PPGAPDS systems

\begin{tabular}{|c|c|c|c|c|}
\hline System & PPG2APDS & PPG5APDS & PPG10APDS & PPG20APDS \\
\hline$D_{\mathrm{p}}[\mathrm{nm}]$ & $162 \pm 12$ & $98 \pm 3$ & $74 \pm 3$ & $35 \pm 4$ \\
\hline
\end{tabular}

monomers into the polymer structure can be confirmed.

Regarding the water dispersion features, the influence of the incorporation of the aromatic disulfide healing agents into the dispersion stability was determined. Table 2 summarizes the particle size values for all formulations, where the introduction of the healing agent in PPGAPDS systems led to a decrease in the polymer particle size.

According to the results, it seems that the interaction of alkoxysilane groups located in the polymer chain ends had a predominant effect in the polymer particle size in water [21, 25, 31]. Even so, all formulations were stable for at least one week.

\subsection{Film forming process}

During the film forming process of PPGAPDS samples, alkoxysilane condensation occurred and consequently the material crosslinked. This process was mainly influenced by the amount of N-bAPTMS monomer in the sample. In order to determine the influence of such a factor in the crosslinking capabilities of these materials, polymer films of different formulations were obtained by crosslinking the samples at room temperature for at least one week and their gel content was calculated by Soxhlet extraction in THF. As can be observed in Figure 4, the gel fraction increased as the amount of alkoxysilane in the sample increased, reaching values higher than $75 \%$ for samples containing more than $10 \mathrm{wt} \%$ of N-bAPTMS. According to the formulations summarized in Table 2,

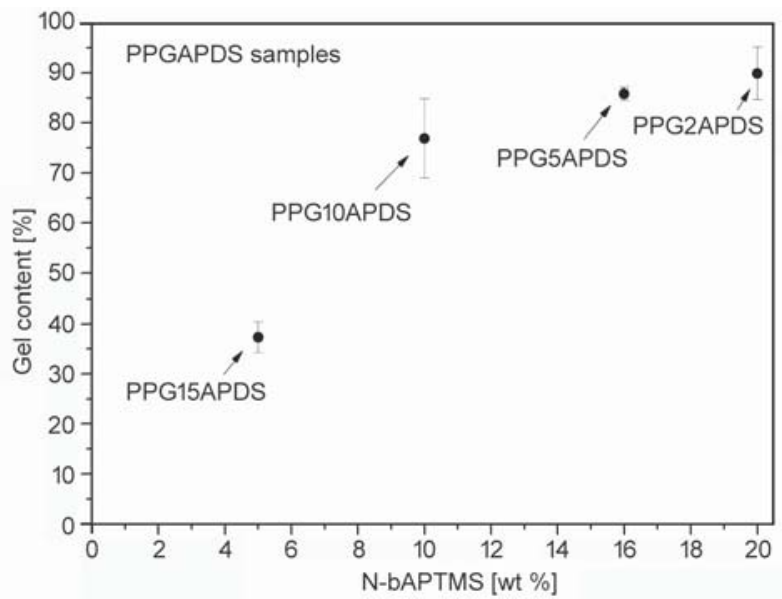

Figure 4. Gel content values for PPGAPDS samples containing different amounts of N-bAPTMS

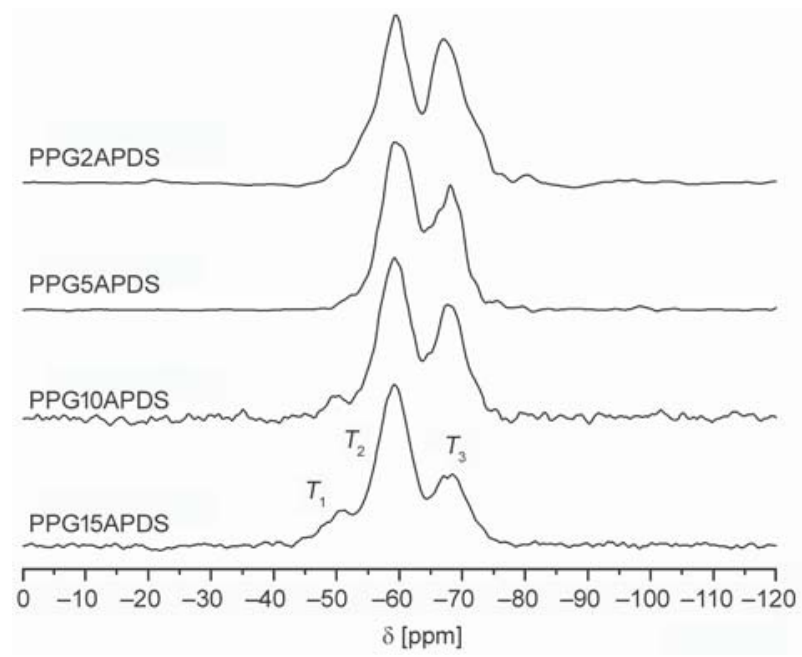

Figure 5. ${ }^{29} \mathrm{Si}-\mathrm{NMR}$ spectra of different PPGAPDS samples

materials with higher gel content values were those with lower APDS concentrations, which suggested that the crosslinking density increased when decreasing the molecular weight of the samples. Therefore, a balance between the crosslinking degree and the healing agent amount should be reached. The crosslinking behavior of these samples was also analyzed by solid state ${ }^{29} \mathrm{Si}-\mathrm{NMR}$ spectroscopy. Following the nomenclature proposed by Lippmaa and coworkers, silicon atoms of N-bAPTMS moieties were classified as $T$ species $\left(T_{1}, T_{2}\right.$ and $T_{3}$ species) [32,33]. The obtained spectra (Figure 5) depict the influence of N-bAPTMS concentration on the sample condensation degree.

Although all samples presented lines corresponding to $T_{2}$ and $T_{3}$ condensed species, additional signals corresponding to $T_{1}$ structures appeared in the spectra of PPG15APDS and PPG10APDS samples. However, the intensity of this $T_{1}$ line decreased as N-bAPTMS concentration in the sample increased while the intensity of the more condensed species $\left(T_{3}\right)$ increased. These results are in good agreement with the corresponding gel content values for these systems. Samples containing higher amounts of N-bAPTMS gave rise to signals attributable to highly condensed structures in their ${ }^{29} \mathrm{Si}-\mathrm{NMR}$ spectra and also high gel fractions. In contrast, the presence of $T_{1}$ species in PPG15APDS indicated the higher amount of uncondensed alkoxysilane species which were responsible for the low gel fraction values.

The formation of silica networks by alkoxysilane condensation is reflected in the morphology that these materials displayed. As a consequence of the intrinsic incompatibility between hard and soft segments as well as the different chemical nature of the 


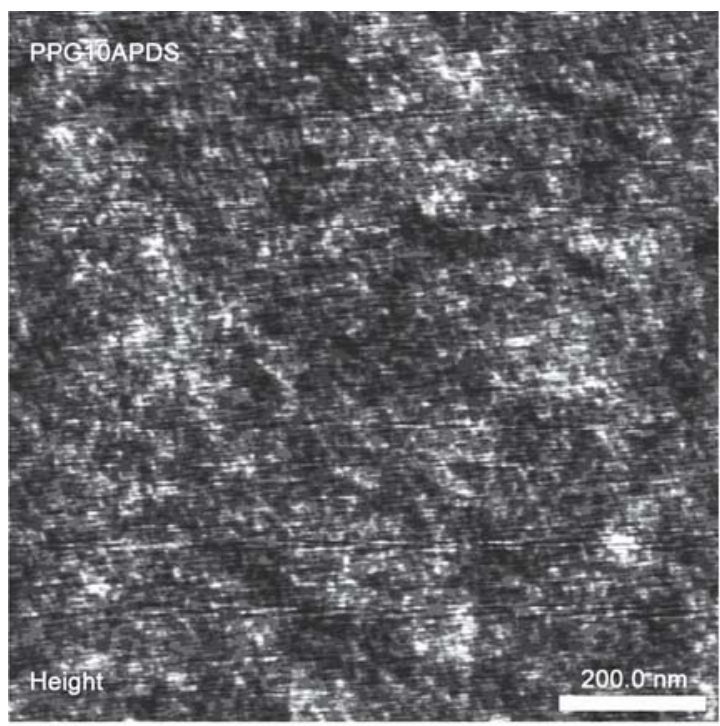

a)

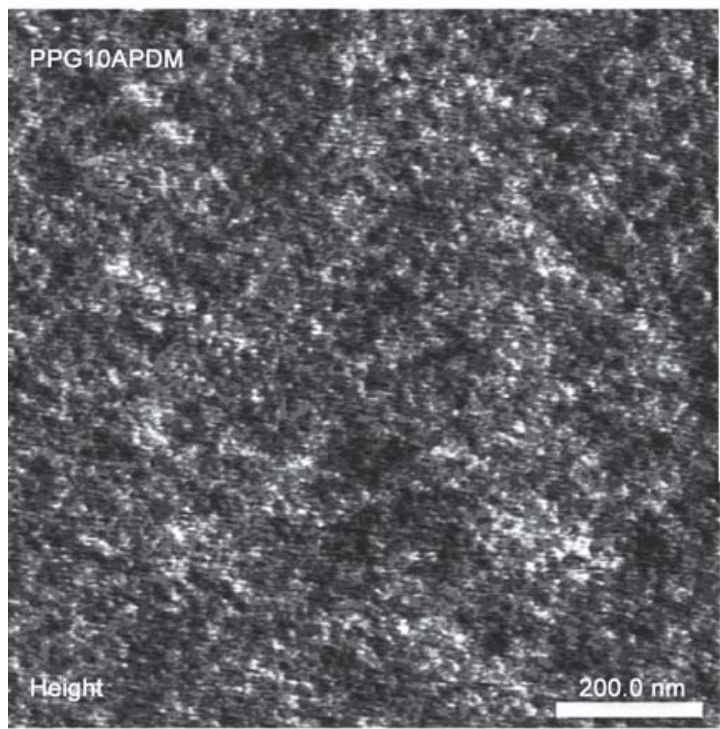

c)

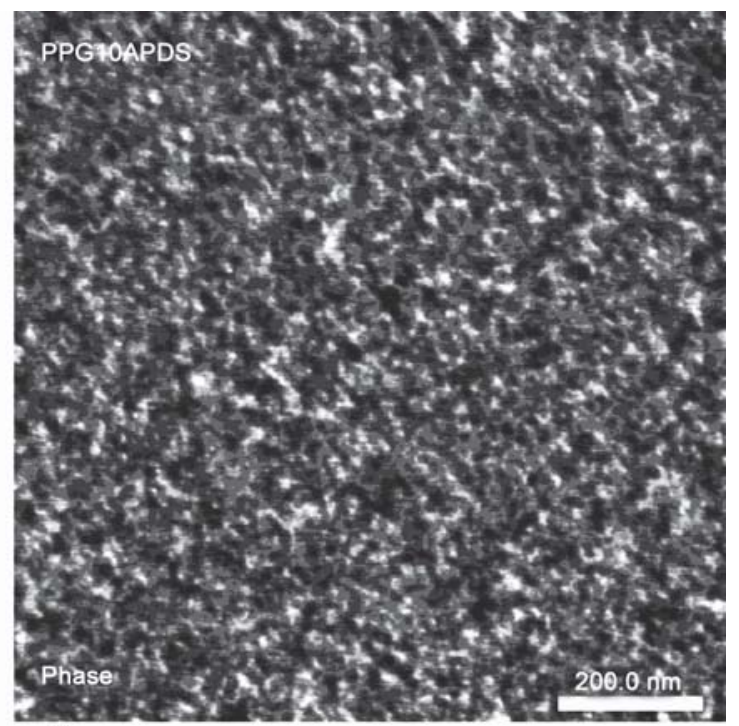

b)

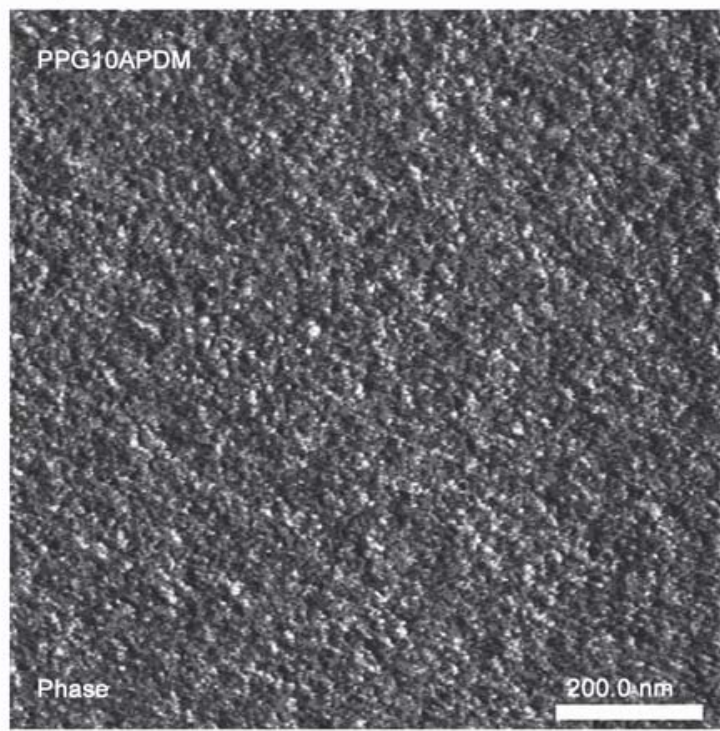

d)

Figure 6. Height (a, c) and phase (b, d) AFM images for PPG10APDS samples (a, b) and PPG10APDM (c, d)

organic and inorganic moieties, these materials tended to form separated domains. However, APDS moieties also self-aggregate via urea bond formation [23]. This specific morphology, observed in the AFM images of Figure 6, can be related both to the inorganic domains formed by condensation of the alkoxysilane precursors or the phase separation of aromatic disulfide moieties [34-37]. From the phase image it was not possible to differentiate the hard segments formed by silicon-oxygen networks from those corresponding to the aromatic disulfide moieties. Nevertheless, taking into consideration the difference in nature between silica domains and the polyurethane structure, it seems more reasonable to associate these regions to silicon-oxygen domains. Even so, the phase separation of aromatic disulfide moieties cannot be discarded.

\subsection{Evaluation of the healing properties}

The disulfide methatesis reaction is on the basis of the healing properties of APDS systems. As represented in Figure 7a, these structures are able to interchange their side groups according to a dynamic equilibrium. When the damage occurs, see Figure $7 \mathrm{~b}$, these disulfide moieties are able to recombine, promoting the generation of new linkages in the damaged surfaces and leading to material reparation.

Polymer films were prepared by casting PPGAPDS water dispersions onto Teflon moulds for one week to allow the crosslinking process to take place. Once the films were obtained, their healing ability was qualitatively evaluated according to the following procedure: samples were cut into two separate parts and finally the recovery of the structural integrity was promoted by putting both sides of the fracture in 


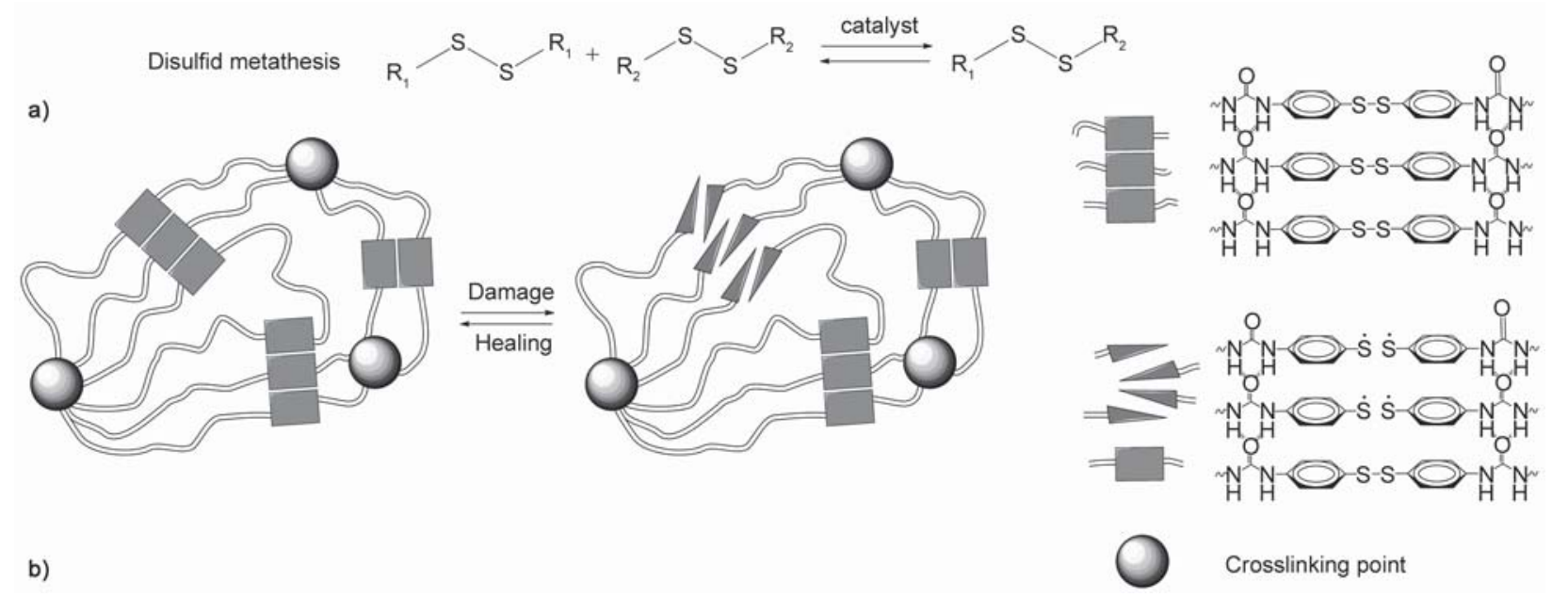

Figure 7. Disulfide methatesis reaction (a) and the proposed healing mechanism by means of aromatic disulfide metathesis (b)
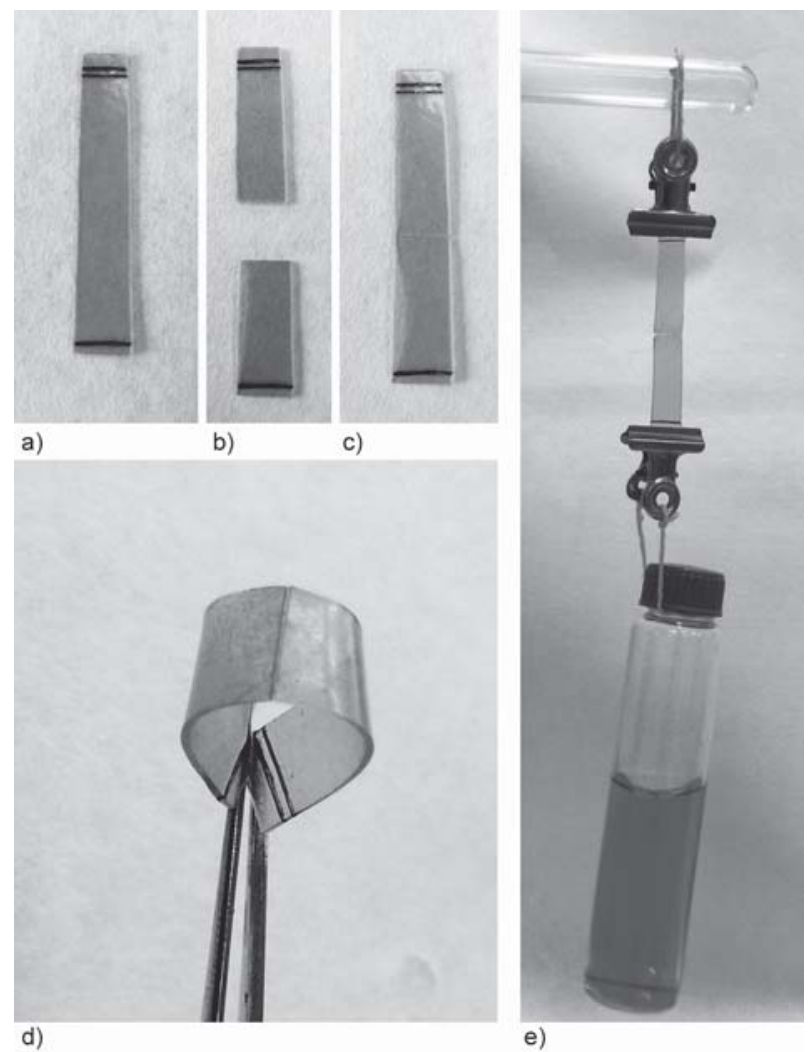

Figure 8. Restoration steps for PPG10APDS sample (pristine (a), cut (b) and healed (c) material). Bending (d) and tensile (e) properties of the healed material as a proof of the restoration

close contact for a healing time of two hours. This procedure was performed immediately after the cut in order to avoid the passivation of the generated surface. As shown in Figure 8, materials were able to recover their structural integrity, to bend and support an arbitrary load without breaking, which proved their autonomic healing properties. However, samples containing low amounts of aromatic disulfide moieties (PPG2APDS) were unable to repair the fracture.
The reparation mechanism in aromatic disulfide based systems is described according to the disulfide metathesis reaction and a contribution of the hydrogen bonds formed between the urea groups [21, 38]. In order to evaluate the extent of the contribution of hydrogen bonding to the healing process, samples containing 4,4'-ethylenedianiline (PPG5APDM and PPG10APDM systems), and therefore without S-S linkages, were qualitatively analyzed following the same procedure. In that case, the PPGAPDM systems showed healing capacity, which confirmed that, in addition to the disulfide metathesis reaction, the presence of hydrogen bonds played a role in the reparation process. Its influece will be discussed in the mecanical characterization of the repaired materials. The mechanical characterization as well as the recovery of the structural integrity of the healable waterborne polyurethanes was carried out by strainstress tests. Firstly, the mechanichal properties of the undamaged materials were determined. Test specimens were obtained by die-cutting about $0.5 \mathrm{~mm}$ thick polymer films in dumbbell-shape. Although the amount of the alkoxysilane precursor (N-bAPTMS) in the synthesized materials ranged from 5 to $20 \mathrm{wt} \%$, the specimens corresponding to the sample containing $5 \mathrm{wt} \%$ of N-bAPTMS (PPG15APDS sample) did not show sufficient integrity to be tested. The obtained stress-strain plots of the remainder of the PPGAPDS samples are presented in Figure 9.

As the N-bAPTMS amount in the sample increased, the elongation at break of the samples decreased while Young's modulus increased. Furthermore, the systems containing $20 \mathrm{wt} \%$ of alkoxysilane groups (PPG2APDS) displayed non elastomeric behavior showing a Yield point at low deformation values. 


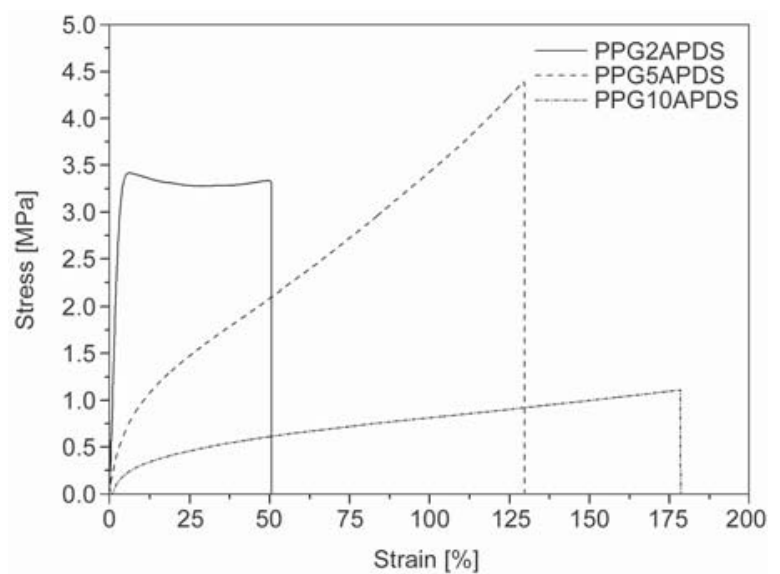

Figure 9. Stress-strain curves for PPGAPDS samples containing different amounts of N-bAPTMS

This fact, together with the low amount of APDS in these systems, can be responsible for the absence of reparation after the fracture of the material, as it did not present enough mobility to permit material diffusion after the fracture.

The quantitative analysis of healing ability was performed by cutting dumbbell shaped specimens and repairing the fracture by putting the generated surfaces in close contact at room temperature $\left(25^{\circ} \mathrm{C}\right)$. In order to determine the time needed to achieve maximum reparation, the repaired specimens were tested after different healing times. This experiment was carried out for both PPG10APDS and PPG10APDM systems, which differred in the monomer used in their synthesis: aromatic disulfide in the case of PPG10APDS and 4,4'-ethylenedianiline for PPG10APDM. Thus, the influence of the hydrogen bonding in the healing process was also analyzed.

From the data of strain-stress tests, the healing efficiency $(\eta)$ of the process was calculated at different times $[39,40]$. This parameter expresses the effectiveness of the healing process and it is defined as the ratio of a physical property for both the healed and the undamaged materials, according to Equation (1):

$\eta=\frac{P_{\text {healed }}}{P_{\text {virgin }}} \cdot 100$

where $P$ is the physical property of interest.

Given the elastomeric character of the samples, the healing efficiency of the process was evaluated using the elongation at break as the physical property of interest. In Figure 10 the evolution of the healing efficiency, calculated both as the elongation at break and material toughness as a function of time is represented.

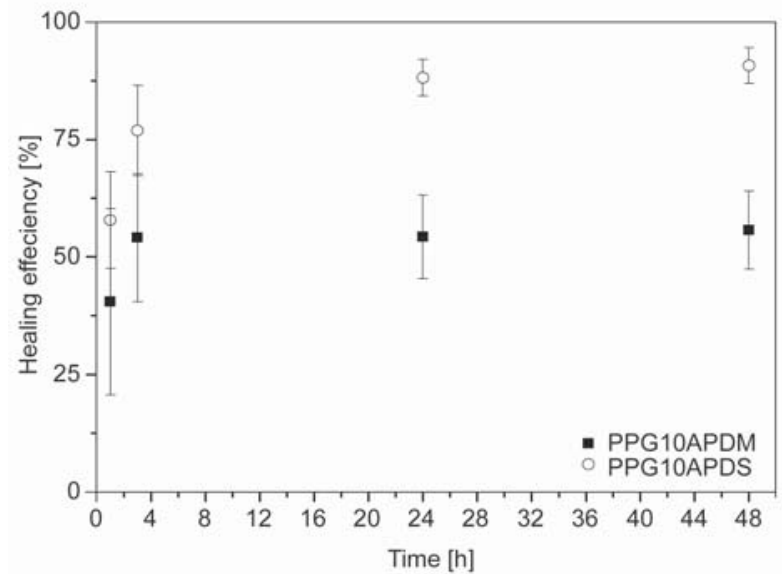

Figure 10. Evolution of the healing efficiency of the reparation process at different times for samples containing disulfide bridges (PPG10APDS) and dimethylene bridges (PPG10APDM)

As observed, the reparation process for disulfide bridges containing samples (PPG10APDS samples) progressed until it reached a plateau around 24 hours. This time dependent reparation process is in good agreement with the dynamic nature of the disulfide metathesis reaction $[15,34]$. In contrast, the maximum reparation of PPG10APDM samples, those which incorporated dimethylene bridges in the structure, was achieved in the first stages of the reparation, around 3 hours, and remained constant over time. In addition, the healing efficiency values remained lower than those corresponing to aromatic disulfide containing systems. Taking into account that the hydrogen bonds formed between the urea groups are responsible for the reparation of the PPGAPDM systems, their countribution to the total healing of PPGAPDS systems can be calculated around $60 \%$. However, other effects can not be discarded. In addition, the different behavior of PPGAPDS and PPGAPDM systems suggests that the hydogen bonds allowed the reparation in the first stages while, in the case of PPGAPDS samples, the disulfide metathesis reaction is responsible for the acquisition of the final structural integrity $[21,38,41]$.

In addition to the evaluation of the healing time, the influence of consecutive damage-healing cycles on the healing efficiency of the process was also studied. This analysis was restricted to the samples containing 5 and $10 \mathrm{wt} \%$ of the healing monomer, as they were the ones which presented healing characteristics in the qualitative analysis. Thus, the quantitative analysis was performed for three independent healing events by cutting the dumbbell shaped specimens 

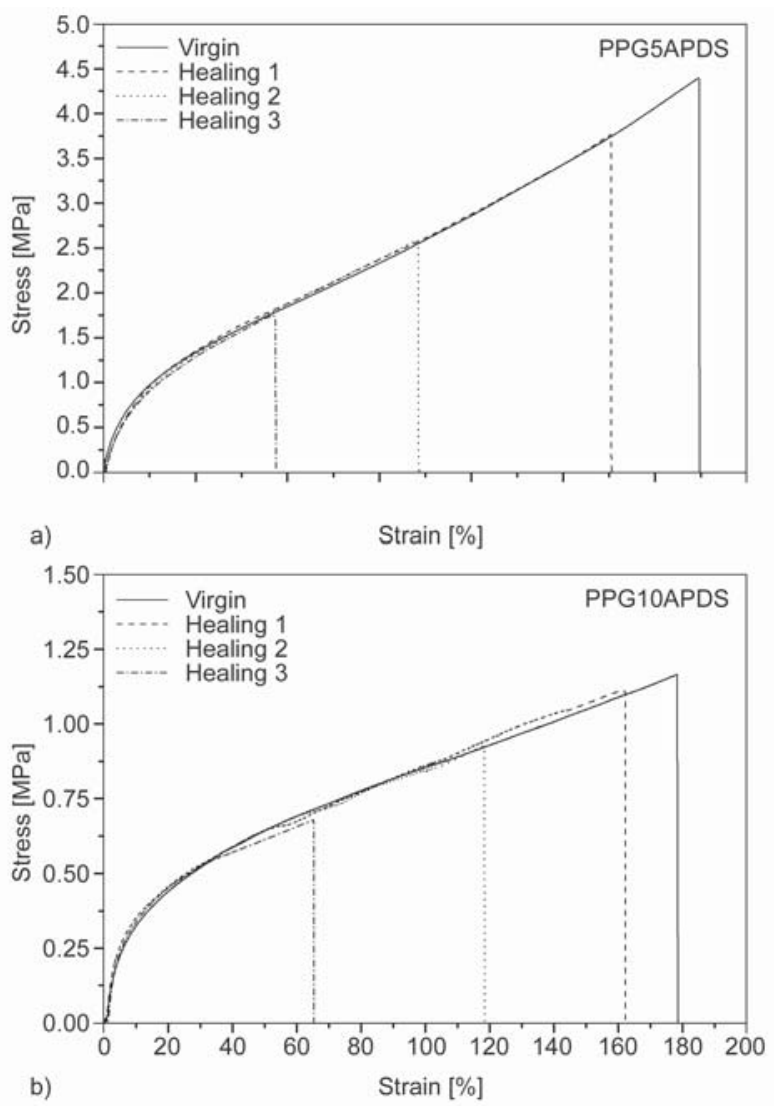

Figure 11. Stress-strain curves corresponding to the different healing events for PPG5APDS (a) and PPG10APDS (b) systems

and repairing the fracture under the appropriate healing conditions after each fracture according to the previous results (a healing time of 48 hours at $25^{\circ} \mathrm{C}$ ). The first cut was made perpendicularly to the applied stress and subsequent fractures were made at $45^{\circ}$ degrees with respect to the first one. Figure 11 shows the stress-strain results for the different healing events. The incorporation of aromatic disulfide moieties into waterborne polyurethane hybrids provided repetitive healing characteristics. After the application of the fracture/healing processes, PPGAPDS samples presented similar physical characteristics to the virgin materials, except for the elongation at break, which decreased as the number of healing events increased. Using Equation (1) the healing efficiency of each process was calculated. In this case, apart from the elongation at break, material toughness was also selected as a physical property of interest and it was calculated as the area under the stress-strain curves. Table 3 summarizes the obtained physical properties as well as healing efficiency values.

The healing efficiency of PPGAPDS, calculated as the recovery of elongation at break and expressed by material toughness, reached values higher than $80 \%$ for both formulations in the first healing event, comparable to those obtained for other autonomic healable systems reported on in literature [13, 42, 43]. However, the effectiveness of the process decreased with the number of healing events.

The healing efficiency increased slightly with aromatic disulfide concentration, mainly in the second and third healing events. Therefore, the healing process seems to be controlled by the dynamic structure concentration. However, the physical properties of the virgin polymer matrix affected the healing ability of the proposed systems. Thus, samples containing low amounts of aromatic disulfide did not present enough polymer chain mobility to permit the material diffusion in the fractured surface, and as a result, they did not present healing characteristics $[10,44]$. In contrast, low amounts of alkoxysilane precursors led to materials with low crosslinking degrees and, therefore, with poor structural integrity. Hence, the healing ability of the proposed polyurethane hybrids is restricted to those materials presenting elastomeric behavior. Even so, as long as the materials behave as elastomers, the incorporation of inorganic precursors has little effect on the healing efficiency

\section{Conclusions}

Aromatic disulfide moieties were successfully introduced into waterborne polyurethanes as chain exten-

Table 3. Healing efficiency of the studied systems as a function of repairing events

\begin{tabular}{|c|c|c|c|c|c|}
\hline \multirow{2}{*}{\multicolumn{2}{|c|}{ Sample }} & \multicolumn{2}{|c|}{ Elongation at break } & \multicolumn{2}{|c|}{ Toughness } \\
\hline & & {$[\%]$} & [Healing \%] & {$\left[\mathbf{J} \cdot \mathbf{m}^{-3}\right]$} & [Healing \%] \\
\hline \multirow{4}{*}{ PPG5APDS } & Virgin & $128 \pm 3$ & - & $3.13 \pm 0.13$ & - \\
\hline & $1^{\text {st }}$ cut & $113 \pm 6$ & $88.6 \pm 3.9$ & $2.58 \pm 0.17$ & $82.4 \pm 7.7$ \\
\hline & $2^{\text {nd }}$ cut & $65 \pm 5$ & $51.0 \pm 8.5$ & $1.03 \pm 0.13$ & $33.1 \pm 12.7$ \\
\hline & $3^{\text {rd }}$ cut & $36 \pm 2$ & $28.6 \pm 4.7$ & $0.42 \pm 0.02$ & $13.6 \pm 5.0$ \\
\hline \multirow{4}{*}{ PPG10APDS } & Virgin & $178 \pm 4$ & - & $1.40 \pm 0.04$ & - \\
\hline & $1^{\text {st }}$ cut & $162 \pm 5$ & $90.7 \pm 3.8$ & $1.22 \pm 0.03$ & $88.0 \pm 3.8$ \\
\hline & $2^{\text {nd }}$ cut & $118 \pm 7$ & $66.1 \pm 6.3$ & $0.76 \pm 0.05$ & $54.6 \pm 7.2$ \\
\hline & $3^{\text {rd }}$ cut & $65 \pm 3$ & $36.4 \pm 5.1$ & $0.32 \pm 0.01$ & $23.0 \pm 4.2$ \\
\hline
\end{tabular}


ders, without interfering in the stability of the corresponding polymer dispersions.

After material crosslinking, via the condensation of alkoxysilane moieties, introduced in the synthesis process, the metathesis reaction of disulfide bridges provided repetitive healing abilities to organic-inorganic polyurethane hybrid films, according to stressstrain measurements. The fracture reparation reached its maximum value after a healing time of 24 hours, after which no significant increment of the healing efficiency was observed. At that time, the healing efficiency, calculated as the ratio of the selected mechanical property of the virgin and repaired materials, reached more than $80 \%$ for the first healing event. However, these values decreased as the number of healing events increased.

Independently of the amount of both healing and crosslinking functionalities, the healing process was observed provided the physical properties of the polymer matrix permitted material diffusion in the fractured surfaces. Therefore, the healing characteristics were restricted to materials with elastomeric behavior. As a conclusion, a balance between the crosslinking degree and the healing ability of the material should be reached in order to obtain healable materials.

\section{Acknowledgements}

The authors acknowledge the University of the Basque Country UPV/EHU (UFI 11/56) and the Basque Government (Ayuda a grupos de investigación del sistema universitario vasco IT 618/13) and the Ministerio de Economía y Competitividad (CTQ2013-4113-R) for the funding received to develop this work.

Technical and Human support provided by MacrobehaviorMesostructure-Nanotechnology and NMR SGiker services of UPV/EHU is also gratefully acknowledged.

\section{References}

[1] Yang Y., Urban M. W.: Self-healing polymeric materials. Chemical Society Reviews, 42, 7446-7467 (2013). https://doi.org/10.1039/c3cs60109a

[2] Ghosh S. K.: Self-healing materials: Fundamentals, design strategies and applications. Wiley-VCH, Weinheim (2009).

[3] Thakur V. K., Kessler M. R.: Self-healing polymer nanocomposite materials: A review. Polymer, 69, 369-383 (2015).

https://doi.org/10.1016/j.polymer.2015.04.086
[4] Yuan Y. C., Yin T., Rong M. Z., Zhang M. Q.: Self healing in polymers and polymer composites. Concepts, realization and outlook: A review. Express Polymer Letters, 2, 238-250 (2008).

https://doi.org/10.3144/expresspolymlett.2008.29

[5] Bang E-K., Lista M., Sforazzini G., Sakai N., Matile S.: Poly(disulfide)s. Chemical Science, 3, 1752-1763 (2012). https://doi.org/10.1039/c2sc20098h

[6] Gyarmati B., Némethy Á., Szilágyi A.: Reversible disulphide formation in polymer networks: A versatile functional group from synthesis to applications. European Polymer Journal, 49, 1268-1286 (2013). https://doi.org/10.1016/j.eurpolymj.2013.03.001

[7] Caraballo R.: Dynamic sulfur chemistry: Screening, evaluation and catalysis. Royal Institute of Technology, Stockholm (2010).

[8] Xiang H. P., Qian H. J., Lu Z. Y., Rong M. Z., Zhang M. $\mathrm{Q}$.: Crack healing and reclaiming of vulcanized rubber by triggering the rearrangement of inherent sulfur crosslinked networks. Green Chemistry, 17, 4315-4325 (2015). https://doi.org/10.1039/c5gc00754b

[9] Urban M. W.: Handbook of stimuli-responsive materials. Wiley-VCH, Weinheim (2011).

[10] Yang Y., Ding X., Urban M. W.: Chemical and physical aspects of self-healing materials. Progress in Polymer Science, 49, 34-59 (2015). https://doi.org/10.1016/j.progpolymsci.2015.06.001

[11] Denissen W., Winne J. M., Du Prez F. E.: Vitrimers: Permanent organic networks with glass-like fluidity. Chemical Science, 7, 30-38 (2016). https://doi.org/10.1039/C5SC02223A

[12] Fiore G. L., Rowan S. J., Weder C.: Optically healable polymers. Chemical Society Reviews, 42, 7278-7288 (2013). https://doi.org/10.1039/c3cs35471g

[13] AbdolahZadeh M., Esteves A. C., van der Zwaag S., Garcia S. J.: Healable dual organic-inorganic crosslinked sol-gel based polymers: Crosslinking density and tetrasulfide content effect. Journal of Polymer Science Part A: Polymer Chemistry, 52, 1953-1961 (2014). https://doi.org/10.1002/pola.27200

[14] Xu W. M., Rong M. Z., Zhang M. Q.: Sunlight driven self-healing, reshaping and recycling of a robust, transparent and yellowing-resistant polymer. Journal of Materials Chemistry A, 4, 10683-10690 (2016). https://doi.org/10.1039/c6ta02662a

[15] Xiang H. P., Rong M. Z., Zhang M. Q.: Self-healing, reshaping, and recycling of vulcanized chloroprene rubber: A case study of multitask cyclic utilization of crosslinked polymer. ACS Sustainable Chemistry and Engineering, 4, 2715-2724 (2016).

https://doi.org/10.1021/acssuschemeng.6b00224 
[16] Michal B. T., Jaye C. A., Spencer E. J., Rowan S. J.: Inherently photohealable and thermal shape-memory polydisulfide networks. ACS Macro Letters, 2, 694 699 (2013).

https://doi.org/10.1021/mz400318m

[17] Lafont U., van Zeijl H., van der Zwaag S.: Influence of cross-linkers on the cohesive and adhesive self-healing ability of polysulfide-based thermosets. ACS Applied Materials and Interfaces, 4, 6280-6288 (2012).

https://doi.org/10.1021/am301879z

[18] Azcune I., Odriozola I.: Aromatic disulfide crosslinks in polymer systems: Self-healing, reprocessability, recyclability and more. European Polymer Journal, 84, 147-160 (2016).

https://doi.org/10.1016/j.eurpolymj.2016.09.023

[19] Belenguer A. M., Friščić T., Day G. M., Sanders J. K. M.: Solid-state dynamic combinatorial chemistry: Reversibility and thermodynamic product selection in covalent mechanosynthesis. Chemical Science, 2, 696700 (2011).

https://doi.org/10.1039/c0sc00533a

[20] Sarma R. J., Otto S., Nitschke J. R.: Disulfides, imines, and metal coordination within a single system: Interplay between three dynamic equilibria. Chemistry-A European Journal, 13, 9542-9546 (2007). https://doi.org/10.1002/chem.200701228

[21] Rekondo A., Martin R., de Luzuriaga A. R., Cabañero G., Grande H. J., Odriozola I.: Catalyst-free room-temperature self-healing elastomers based on aromatic disulfide metathesis. Materials Horizons, 1, 237-240 (2014). https://doi.org/10.1039/C3MH00061C

[22] Martin R., Rekondo A., de Luzuriaga A. R., Santamaria A., Odriozola I.: Mixing the immiscible: Blends of dynamic polymer networks. RSC Advances, 5, 17514 17518 (2015).

https://doi.org/10.1039/C5RA00628G

[23] Martin R., Rekondo A., de Luzuriaga A. R., Cabañero G., Grande H. J., Odriozola I.: The processability of a poly(urea-urethane) elastomer reversibly crosslinked with aromatic disulfide bridges. Journal of Materials Chemistry A: Materials for energy and sustainability, 2, 5710-5715 (2014).

https://doi.org/10.1039/c3ta14927g

[24] Abdolah Zadeh M., van der Zwaag S., Garcia S. J.: Routes to extrinsic and intrinsic self-healing corrosion protective sol-gel coatings: A review. Self-Healing Materials, 1, 1-18 (2013).

https://doi.org/10.2478/shm-2013-0001

[25] Sardon H., Irusta L., Fernández-Berridi M. J., Lansalot M., Bourgeat-Lami E.: Synthesis of room temperature self-curable waterborne hybrid polyurethanes functionalized with (3-aminopropyl)triethoxysilane (APTES). Polymer, 51, 5051-5057 (2010).

https://doi.org/10.1016/j.polymer.2010.08.035
[26] Sardon H., Irusta L., Aguirresarobe R. H., FernándezBerridi M. J.: Polymer/silica nanohybrids by means of tetraethoxysilane sol-gel condensation onto waterborne polyurethane particles. Progress in Organic Coatings, 77, 1436-1442 (2014).

https://doi.org/10.1016/j.porgcoat.2014.04.032

[27] Sardon H., Irusta L., Santamaría P., Fernández-Berridi M. J.: Thermal and mechanical behaviour of self-curable waterborne hybrid polyurethanes functionalized with (3-aminopropyl)triethoxysilane (APTES). Journal of Polymer Research, 19, 9956-9965 (2012). https://doi.org/10.1007/s10965-012-9956-8

[28] Sardon H., Irusta L., Fernández-Berridi M. J., Luna J., Lansalot M., Bourgeat-Lami E.: Waterborne polyurethane dispersions obtained by the acetone process: A study of colloidal features. Journal of Applied Polymer Science, 120, 2054-2062 (2011).

https://doi.org/10.1002/app.33308

[29] Aguirresarobe R. H., Irusta L., Fernández-Berridi M. J.: UV-light responsive waterborne polyurethane based on coumarin: Synthesis and kinetics of reversible chain extension. Journal of Polymer Research, 21, 505-508 (2014).

https://doi.org/10.1007/s10965-014-0505-5

[30] Aguirresarobe R. H., Martin L., Aramburu N., Irusta L., Fernandez-Berridi M. J.: Coumarin based light responsive healable waterborne polyurethanes. Progress in Organic Coatings, 99, 314-321 (2016).

https://doi.org/10.1016/j.porgcoat.2016.06.011

[31] Wang L., Shen Y., Lai X., Li Z., Liu M.: Synthesis and properties of crosslinked waterborne polyurethane. Journal of Polymer Research, 18, 469-476 (2010). https://doi.org/10.1007/s10965-010-9438-9

[32] Lippmaa E., Maegi M., Samoson A., Engelhardt G., Grimmer A. R.: Structural studies of silicates by solidstate high-resolution silicon-29 NMR. Journal of the American Chemical Society, 102, 4889-4893 (1980). https://doi.org/10.1021/ja00535a008

[33] Magi M., Lippmaa E., Samoson A., Engelhardt G., Grimmer A. R.: Solid-state high-resolution silicon-29 chemical shifts in silicates. Journal of Physical Chemistry, 88, 1518-1522 (1984).

https://doi.org/10.1021/j150652a015

[34] Metwalli E., Haines D., Becker O., Conzone S., Pantano C. G.: Surface characterizations of mono-, di-, and tri-aminosilane treated glass substrates. Journal of Colloid and Interface Science, 298, 825-831 (2006). https://doi.org/10.1016/j.jcis.2006.03.045

[35] Guo S. Z., Zhang C., Wang W. Z., Liu T. X.: Preparation and characterization of organic-inorganic hybrid nanomaterials using polyurethane-b-poly[3-(trimethoxysilyl) propyl methacrylate via RAFT polymerization. Express Polymer Letters, 4, 17-25 (2010). https://doi.org/10.3144/expresspolymlett.2010.4 
[36] Garrett J. T., Siedlecki C. A., Runt J.: Microdomain morphology of poly(urethane urea) multiblock copolymers. Macromolecules, 34, 7066-7070 (2001). https://doi.org/10.1021/ma0102114

[37] McLean R. S., Sauer B. B.: Tapping-mode AFM studies using phase detection for resolution of nanophases in segmented polyurethanes and other block copolymers. Macromolecules, 30, 8314-8317 (1997).

https://doi.org/10.1021/ma970350e

[38] Matxain J. M., Asua J. M., Ruipérez F.: Design of new disulfide-based organic compounds for the improvement of self-healing materials. Physical Chemistry Chemical Physics, 18, 1758-1770 (2016).

https://doi.org/10.1039/C5CP06660C

[39] Mauldin T., Kessler M.: Self-healing polymers and composites. International Materials Reviews, 55, $317-$ 346 (2010).

https://doi.org/10.1179/095066010X12646898728408

[40] Blaiszik B. J., Kramer S. L. B., Olugebefola S. C., Moore J. S., Sottos N. R., White S. R.: Self-healing polymers and composites. Annual Review of Material Research, 40, 179-211 (2010).

https://doi.org/10.1146/annurev-matsci-070909-104532
[41] Grande A. M., Bijleveld J. C., Garcia S. J., van der Zwaag S.: A combined fracture mechanical - Rheological study to separate the contributions of hydrogen bonds and disulphide linkages to the healing of poly (urea-urethane) networks. Polymer, 96, 26-34 (2016). https://doi.org/10.1016/j.polymer.2016.05.004

[42] Canadell J., Goossens H., Klumperman B.: Self-healing materials based on disulfide links. Macromolecules, 44, 2536-2541 (2011). https://doi.org/10.1021/ma2001492

[43] Pepels M., Filot I., Klumperman B., Goossens H.: Selfhealing systems based on disulfide-thiol exchange reactions. Polymer Chemistry, 4, 4955-4965 (2013). https://doi.org/10.1039/C3PY00087G

[44] Kim Y. H., Wool R. P.: A theory of healing at a polymer-polymer interface. Macromolecules, 16, 11151120 (1983). https://oi.org/10.1021/ma00241a013 